Network Working Group

Request for Comments: 4619

Category: Standards Track
L. Martini, Ed. Cisco Systems, Inc. C. Kawa, Ed. Oz Communications A. Malis, Ed. Tellabs

September 2006

\title{
Encapsulation Methods for Transport of Frame Relay over Multiprotocol Label Switching (MPLS) Networks
}

Status of This Memo

This document specifies an Internet standards track protocol for the Internet community, and requests discussion and suggestions for improvements. Please refer to the current edition of the "Internet Official Protocol standards" (STD 1) for the standardization state and status of this protocol. Distribution of this memo is unlimited.

Copyright Notice

Copyright (C) The Internet Society (2006).

Abstract

A frame relay pseudowire is a mechanism that exists between a provider's edge network nodes and that supports as faithfully as possible frame relay services over an MPLS packet switched network (PSN). This document describes the detailed encapsulation necessary to transport frame relay packets over an MPLS network. 
Table of Contents

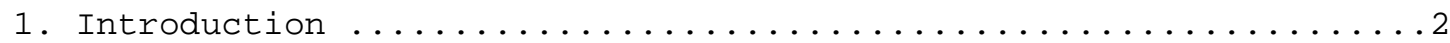

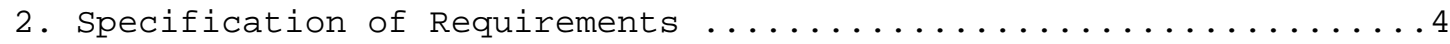

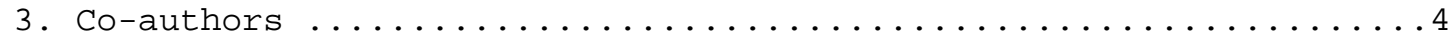

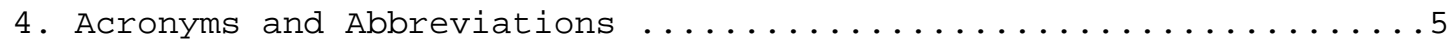

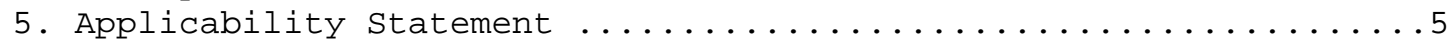

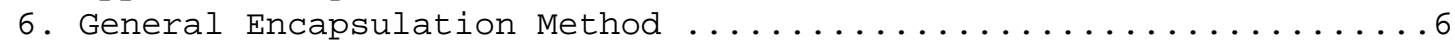

7. Frame Relay over MPLS PSN for the one-to-one Mode ...........7

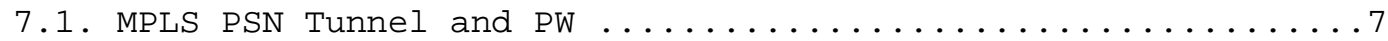

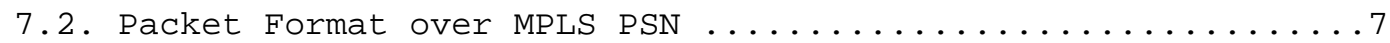

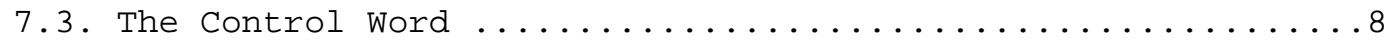

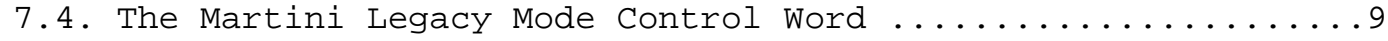

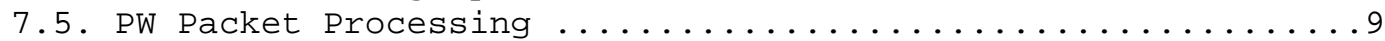

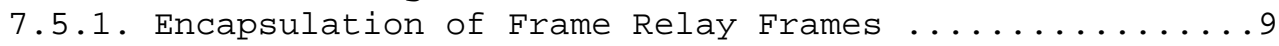

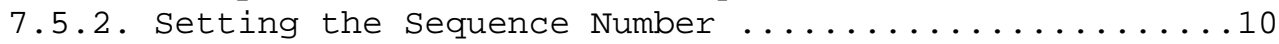

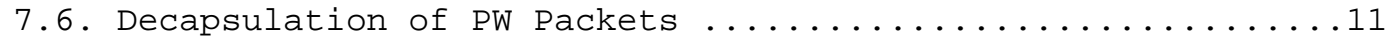

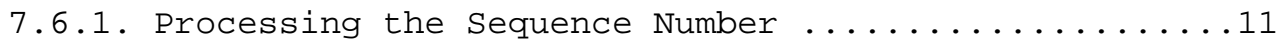

7.6.2. Processing of the Length Field by the Receiver ....11

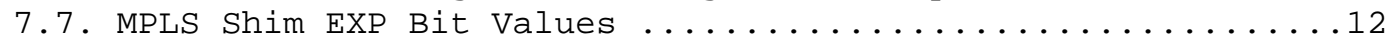

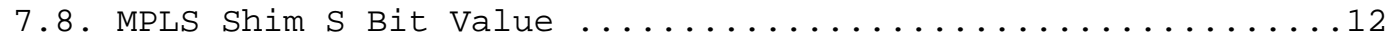

7.9. Control Plane Details for Frame Relay Service ..........12

7.9.1. Frame Relay Specific Interface Parameter Sub-TLV ...12

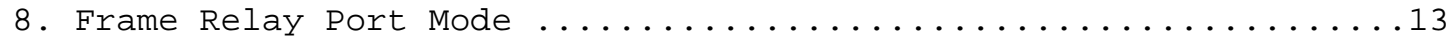

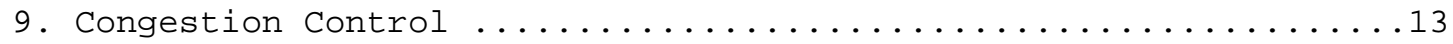

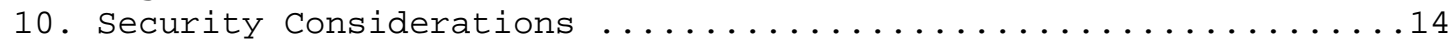

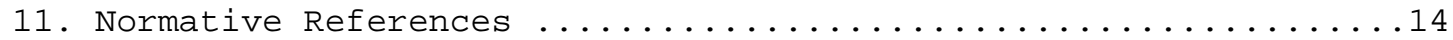

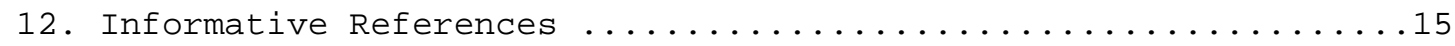

\section{Introduction}

In an MPLS or IP network, it is possible to use control protocols such as those specified in [RFC4447] to set up "pseudowires" (PWs) that carry the Protocol Data Units of layer 2 protocols across the network. A number of these emulated PWs may be carried in a single tunnel. The main functions required to support frame relay PW by a Provider Edge ( $\mathrm{PE})$ include:

- encapsulation of frame relay specific information in a suitable pseudowire (PW) packet;

- transfer of a PW packet across an MPLS network for delivery to a peer $\mathrm{PE}$;

- extraction of frame relay specific information from a PW packet by the remote peer $\mathrm{PE}$; 
- regeneration of native frame relay frames for forwarding across an egress port of the remote peer $\mathrm{PE}$; and

- execution of any other operations as required to support frame relay service.

This document specifies the encapsulation for the emulated frame relay VC over an MPLS PSN. Although different layer 2 protocols require different information to be carried in this encapsulation, an attempt has been made to make the encapsulation as common as possible for all layer 2 protocols. Other layer 2 protocols are described in separate documents. [ATM] [RFC4448] [RFC4618]

The following figure describes the reference models that are derived from [RFC3985] to support the frame relay PW emulated services.

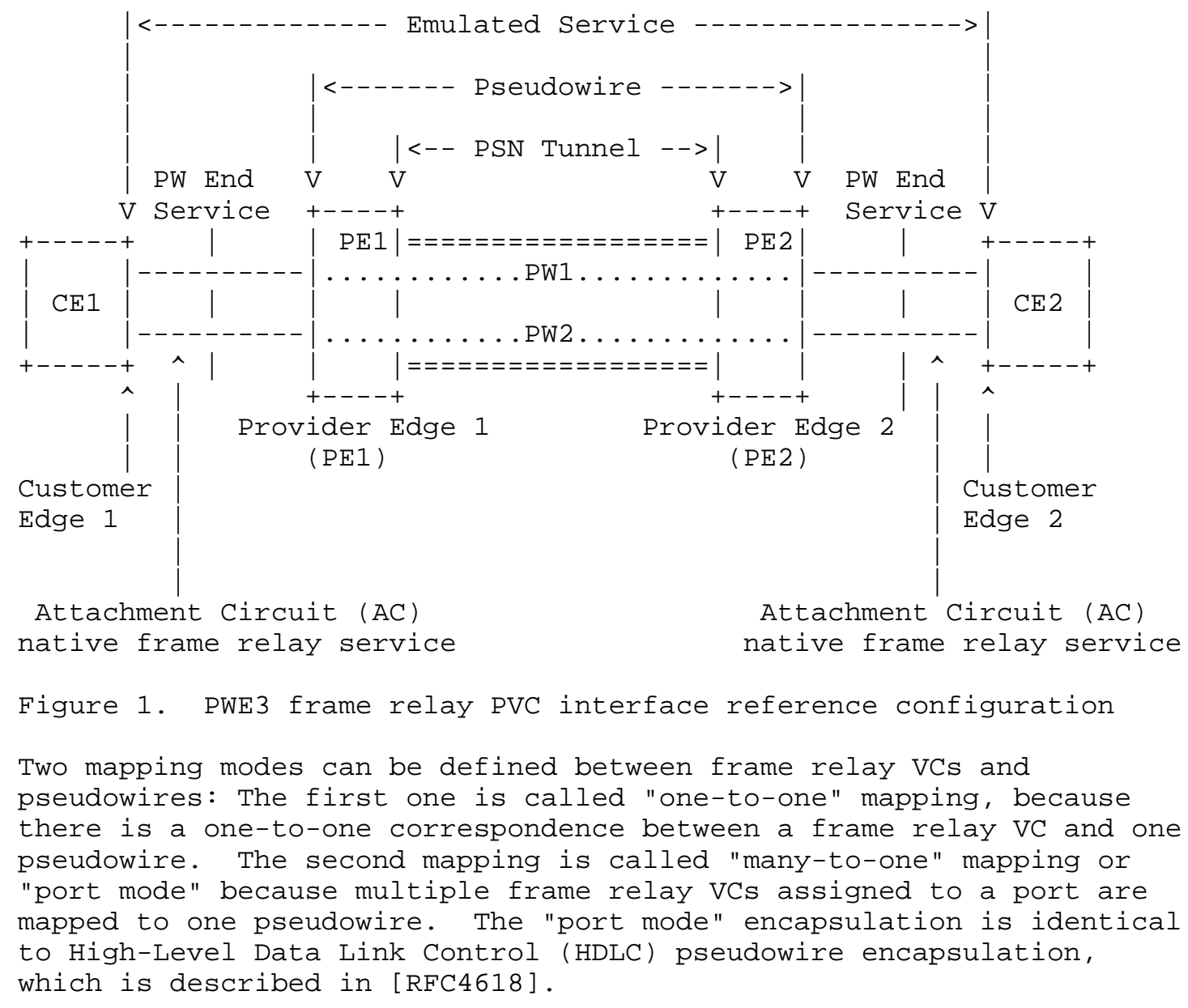


2. Specification of Requirements

The key words "MUST", "MUST NOT", "REQUIRED", "SHALL", "SHALL NOT", "SHOULD", "SHOULD NOT", "RECOMMENDED", "MAY", and "OPTIONAL" in this document are to be interpreted as described in RFC 2119.

Below are the definitions for the terms used throughout the document. PWE3 definitions can be found in [RFC3916, RFC3985]. This section defines terms specific to frame relay.

- Forward direction

The forward direction is the direction taken by the frame being forwarded.

- Backward direction

In frame relay, it is the direction opposite to the direction taken by a frame being forwarded (see also forward direction).

3. Co-authors

The following are co-authors of this document:

Nasser El-Aawar

Eric C. Rosen

Daniel Tappan

Thomas K. Johnson

Kireeti Kompella

Steve Vogelsang

Vinai Sirkay

Ravi Bhat

Nishit Vasavada

Giles Heron

Dimitri Stratton Vlachos

Chris Liljenstolpe

Prayson Pate
Level 3 Communications, LLC

Cisco Systems

Cisco Systems

Litchfield Communications

Juniper Networks, Inc.

Laurel Networks, Inc.

Reliance Infocomm

Nokia

Nokia

Tellabs

Mazu Networks, Inc.

Cable \& Wireless

Overture Networks, Inc 
4. Acronyms and Abbreviations

$\begin{array}{ll}\text { BECN } & \text { Backward Explicit Congestion Notification } \\ \text { CE } & \text { Customer Edge } \\ \text { C/R } & \text { Command/Response } \\ \text { DE } & \text { Discard Eligibility } \\ \text { DLCI } & \text { Data Link Connection Identifier } \\ \text { FCS } & \text { Frame Check Sequence } \\ \text { FECN } & \text { Forward Explicit Congestion Notification } \\ \text { FR } & \text { Frame Relay } \\ \text { LSP } & \text { Label Switched Path } \\ \text { LSR } & \text { Label Switching Router } \\ \text { MPLS } & \text { Multiprotocol Label Switching } \\ \text { MTU } & \text { Maximum Transfer Unit } \\ \text { NNI } & \text { Network-Network Interface } \\ \text { PE } & \text { Provider Edge } \\ \text { PSN } & \text { Packet Switched Network } \\ \text { PW } & \text { Pseudowire } \\ \text { PWE3 } & \text { Pseudowire Emulation Edge to Edge } \\ \text { POS } & \text { Packet over SoNET/SDH } \\ \text { PVC } & \text { Permanent Virtual Circuit } \\ \text { QOS } & \text { Quality of Service } \\ \text { SVC } & \text { Switched Virtual Circuit } \\ \text { UNI } & \text { User-Network Interface } \\ \text { VC } & \text { Virtual Circuit } \\ & \end{array}$

5. Applicability statement

Frame relay over PW service is not intended to emulate the traditional frame relay service perfectly, but it can be used for applications that need frame relay transport service.

The following are notable differences between traditional frame relay service and the protocol described in this document:

- Frame ordering can be preserved using the OPTIONAL sequence field in the control word; however, implementations are not required to support this feature.

- The Quality of Service model for traditional frame relay can be emulated; however, this is outside the scope of this document.

- A Frame relay port mode PW does not process any frame relay status messages or alarms as described in [Q922] [Q933]

- The frame relay BECN and FECN bit are transparent to the MPLS network and cannot reflect the status of the MPLS network. 
- Support for frame relay SVC and Switched Permanent Virtual Circuit (SPVC) is outside the scope of this document.

- Frame relay Local Management Interface (LMI) is terminated locally in the $\mathrm{PE}$ connected to the frame relay attachment circuit.

- The support of PVC link integrity check is outside the scope of this document.

6. General Encapsulation Method

The general frame relay pseudowire packet format for carrying frame relay information (user's payload and frame relay control

information) between two $\mathrm{PEs}$ is shown in Figure 2.

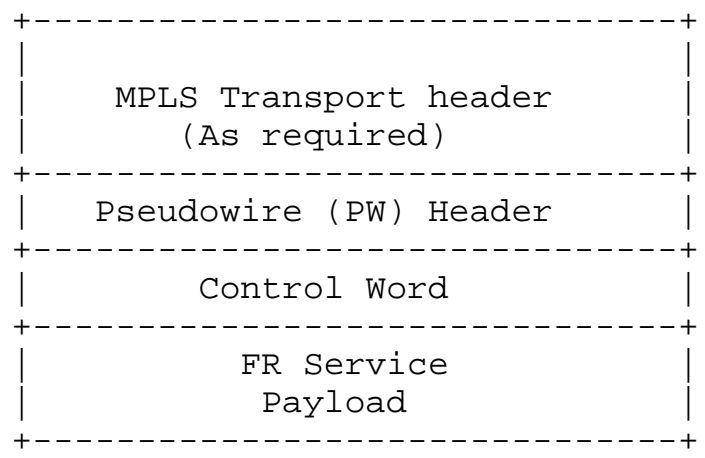

Figure 2. General format of frame relay encapsulation over PSN

The PW packet consists of the following fields: Control word and Payload, preceded by the MPLS Transport and pseudowire header. The meaning of the different fields is as follows:

-i. MPLS Transport header is specific to the MPLS network. This header is used to switch the PW packet through the MPLS core.

-ii. PW header contains an identifier for multiplexing PWs within an MPLS tunnel.

-iii. Control Word contains protocol control information for providing a frame relay service. Its structure is provided in the following sections.

-iv. The content of the frame relay service payload field depends on the mapping mode. In general it contains the layer 2 frame relay frame. 
7. Frame Relay over MPLS PSN for the One-to-One Mode

7.1. MPLS PSN Tunnel and PW

MPLS label switched paths (LSPS) called "MPLS Tunnels" are used between PEs and are used within the MPLS core network to forward PW packets. An MPLS tunnel corresponds to "PSN Tunnel" of Figure 1.

Several PWs may be nested inside one MPLS tunnel. Each PW carries the traffic of a single frame relay VC. In this case, the PW header is an MPLS label called the PW label.

\subsection{Packet Format over MPLS PSN}

For the one-to-one mapping mode for frame relay over an MPLS network, the PW packet format is as shown in Figure 3.

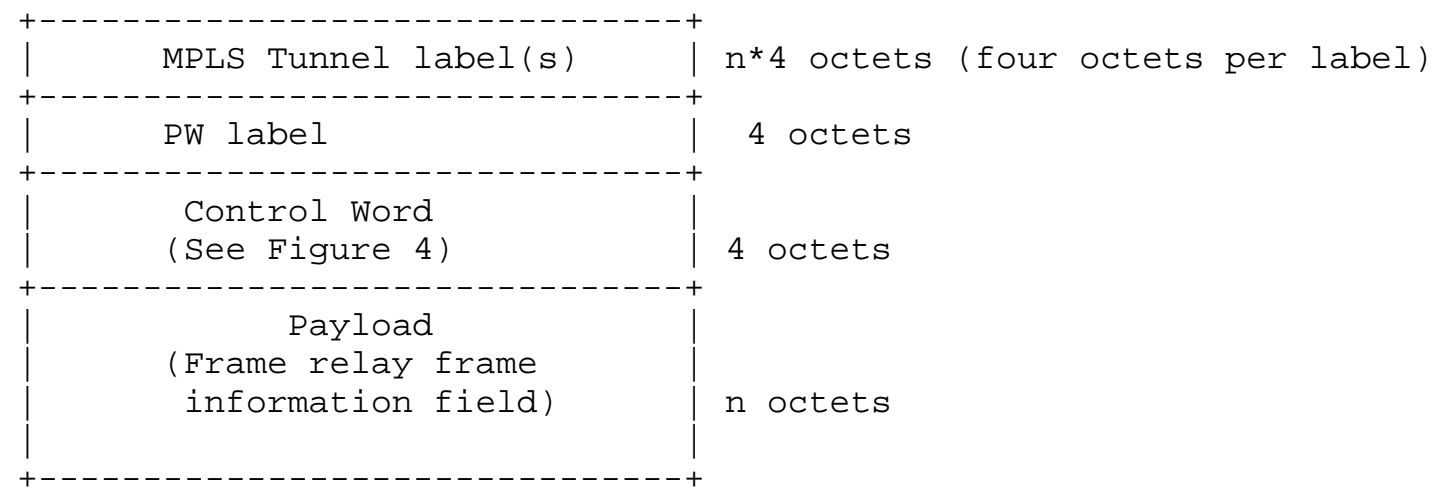

Figure 3. Frame Relay over MPLS PSN Packet for the One-to-One Mapping

The meaning of the different fields is as follows:

- MPLS Tunnel label(s)

The MPLS Tunnel label(s) corresponds to the MPLS transport header of Figure 2. The label(s) is/are used by MPLS LSRs to forward a PW packet from one $\mathrm{PE}$ to the other.

- PW Label

The PW label identifies one PW (i.e., one LSP) assigned to a frame relay $\mathrm{VC}$ in one direction. It corresponds to the PW header of Figure 2. Together the MPLS Tunnel label(s) and PW label form an MPLS label stack [RFC3032]. 
- Control Word

The Control Word contains protocol control information. Its structure is shown in Figure 4.

- Payload

The payload field corresponds to X.36/X.76 frame relay frame information field with the following components removed: bit/byte stuffing, frame relay header, and FCS. It is RECOMMENDED to support a frame size of at least 1600 bytes. The maximum length of the payload field MUST be agreed upon by the two PEs. This can be achieved by using the MTU interface parameter when the PW is established. [RFC4447]

\subsection{The Control Word}

The control word defined below is REQUIRED for frame relay one-to-one mode. The control word carries certain frame relay specific information that is necessary to regenerate the frame relay frame on the egress PE. Additionally, the control word also carries a sequence number that can be used to preserve sequentiality when carrying frame relay over an MPLS network. Its structure is as follows:

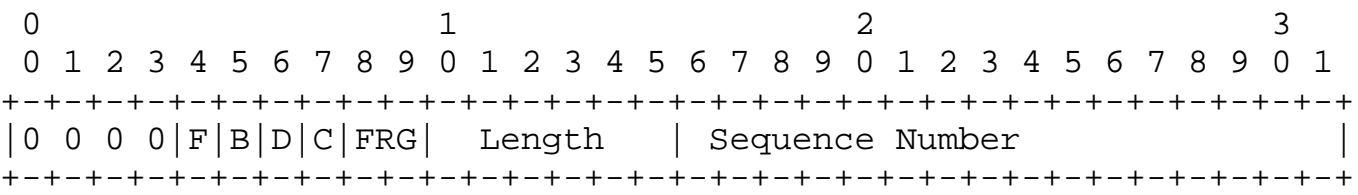

Figure 4. Control Word structure for the one-to-one mapping mode The meaning of the Control Word fields (Figure 4) is as follows (see also [X36 and X76] for frame relay bits):

- Bits 0 to 3

In the above diagram, the first 4 bits MUST be set to 0 to indicate $\mathrm{PW}$ data.

- F (bit 4) FR FECN (Forward Explicit Congestion Notification) bit.

- B (bit 5) FR BECN (Backward Explicit Congestion Notification) bit.

- D (bit 6) FR DE bit (Discard Eligibility) bit.

- C (bit 7) FR frame C/R (Command/Response) bit. 
- FRG (bits 8 and 9): These bits are defined by [RFC4623].

- Length (bits 10 to 15)

If the PW traverses a network link that requires a minimum frame size (a notable example is Ethernet), padding is required to reach its minimum frame size. If the frame's length (defined as the length of the layer 2 payload plus the length of the control word) is less than 64 octets, the length field MUST be set to the PW payload length. Otherwise, the length field MUST be set to zero. The value of the length field, if non-zero, is used to remove the padding characters by the egress $\mathrm{PE}$.

- Sequence number (Bit 16 to 31 )

Sequence numbers provide one possible mechanism to ensure the ordered delivery of PW packets. The processing of the sequence number field is OPTIONAL. The sequence number space is a 16-bit unsigned circular space. The sequence number value 0 is used to indicate that the sequence number check algorithm is not used.

\subsection{The Martini Legacy Mode Control Word}

For backward compatibility to existing implementations, the following version of the control word is defined as the "martini mode CW" for frame relay.

$\begin{array}{llllllllllll}0 & 1 & 2 & 3\end{array}$

$\begin{array}{llllllllllllllllllllllllllllllll}0 & 1 & 2 & 3 & 4 & 5 & 6 & 7 & 8 & 9 & 0 & 1 & 2 & 3 & 4 & 5 & 6 & 7 & 8 & 9 & 0 & 1 & 2 & 3 & 4 & 5 & 6 & 7 & 8 & 9 & 0 & 1\end{array}$

$+-+-+-+-+-+-+-+-+-+-+-+-+-+-+-+-+-+-+-+-+-+-+-+-+-+-+-+-+-+-+-+-+$

\begin{tabular}{|llll}
0 & 0 & 0 & 0
\end{tabular}$|\mathrm{~B}| \mathrm{F}|\mathrm{D}| \mathrm{C}|\mathrm{FRG}|$ Length | Sequence Number

$+-+-+-+-+-+-+-+-+-+-+-+-+-+-+-+-+-+-+-+-+-+-+-+-+-+-+-+-+-+-+-+-+$

Figure 5. Control Word structure for the frame relay martini mode Note that the "B" and "F" bits are reversed.

This control word format is used for PW type "Frame Relay DLCI ( Martini Mode )"

\subsection{PW Packet Processing}

\subsubsection{Encapsulation of Frame Relay Frames}

The encapsulation process of a frame relay frame is initiated when a $\mathrm{PE}$ receives a frame relay frame from one of its frame relay UNI or NNI [FRF1] [FRF2] interfaces. The PE generates the following fields 
of the control word from the corresponding fields of the frame relay frame as follows:

- Command/Response (C/R or C) bit: The C bit is copied unchanged in the PW Control Word.

- The DE bit of the frame relay frame is copied into the D bit field. However, if the $\mathrm{D}$ bit is not already set, it MAY be set as a result of ingress frame policing. If it is not already set by the copy operation, setting of this bit by a PE is OPTIONAL. The PE MUST NOT clear this bit (set it to 0 if it was received with the value of 1 ).

- The FECN bit of the frame relay frame is copied into the $\mathrm{F}$ bit field. However, if the $\mathrm{F}$ bit is not already set, it MAY be set to reflect a congestion situation detected by the $\mathrm{PE}$. If it is not already set by the copy operation, setting of this bit by a PE is OPTIONAL. The PE MUST NOT clear this bit (set it to 0 if it was received with the value of 1 )

- The BECN bit of the frame relay frame is copied into the B bit field. However, if the $B$ bit is not already set, it MAY be set to reflect a congestion situation detected by the PE. If it is not already set by the copy operation, setting of this bit by a PE is OPTIONAL. The PE MUST NOT clear this bit (set it to 0 if it was received with the value of 1 ).

- If the PW packet length (defined as the length of the payload plus the length of the control word) is less than 64 octets, the length field MUST be set to the packet's length. Otherwise, the length field MUST be set to zero.

- The sequence number field is processed if the PW uses sequence numbers. [RFC4385]

- The payload of the PW packet is the contents of ITU-T Recommendations X.36/X.76 [X36] [X76] frame relay frame information field stripped from any bit or byte stuffing.

\subsubsection{Setting the Sequence Number}

For a given PW and a pair of routers PE1 and PE2, if PE1 supports packet sequencing, then the procedures in [RFC4385], Section 4.1, MUST be followed. 


\subsection{Decapsulation of PW Packets}

When a PE receives a PW packet, it processes the different fields of the control word in order to decapsulate the frame relay frame for transmission to a $\mathrm{CE}$ on a frame relay UNI or NNI. The PE performs the following actions (not necessarily in the order shown):

- It generates the following frame relay frame header fields from the corresponding fields of the PW packet.

- The C/R bit MUST be copied in the frame relay header.

- The D bit MUST be copied into the frame relay header DE bit.

- The F bit MUST be copied into the frame relay header FECN bit. If the $\mathrm{F}$ bit is set to zero, the FECN bit may be set to one, depending on the congestion state of the PE device in the forward direction. Changing the state of this bit by a PE is OPTIONAL.

- The B bit MUST be copied into the frame relay header BECN bit. If the B bit is set to zero, the BECN bit may be set to one, depending on the congestion state of the PE device in the backward direction. Changing the state of this bit by a PE is OPTIONAL.

- It processes the length and sequence field, the details of which are in the following sub-sections.

- It copies the frame relay information field from the contents of the PW packet payload after removing any padding.

Once the above fields of a FR frame have been processed, the standard HDLC operations are performed on the frame relay frame: the HDLC header is added, any bit or byte stuffing is added as required, and the FCS is also appended to the frame. The FR frame is then queued for transmission on the selected frame relay UNI or NNI interface.

7.6.1. Processing the Sequence Number

If a router PE2 supports received sequence number processing, then the procedures in [RFC4385], Section 4.2, MUST be used.

7.6.2. Processing of the Length Field by the Receiver

Any padding octet, if present, in the payload field of a PW packet received MUST be removed before forwarding the data.

- If the Length field is set to zero, then there are no padding octets following the payload field. 
- Otherwise, if the payload is longer, then the length specified in the control word padding characters are removed according to the length field.

\subsection{MPLS Shim EXP Bit Values}

If it is desired to carry Quality of Service information, the Quality of Service information SHOULD be represented in the Experimental Use Bits (EXP) field of the PW MPLS label [RFC3032]. If more than one MPLS label is imposed by the ingress LSR, the EXP field of any labels higher in the stack SHOULD also carry the same value.

\subsection{MPLS Shim S Bit Value}

The ingress LSR, PEl, MUST set the $S$ bit of the PW label to a value of 1 to denote that the PW label is at the bottom of the stack.

\subsection{Control Plane Details for Frame Relay Service}

The PE MUST provide frame relay PVC status signaling to the frame relay network. If the $\mathrm{PE}$ detects a service-affecting condition for a particular DLCI, as defined in [Q933] Q.933, Annex A.5, sited in IA FRF1.1, the PE MUST communicate to the remote PE the status of the $P W$ that corresponds to the frame relay DLCI status. The Egress PE SHOULD generate the corresponding errors and alarms as defined in [Q922] [Q933] on the egress Frame relay PVC.

There are two frame relay flags to control word bit mappings described below. The legacy bit ordering scheme will be used for a PW of type 0x0001, "Frame Relay DLCI (Martini Mode)", and the new bit ordering scheme will be used for a PW of type 0x0019, "Frame Relay DLCI". The IANA allocation registry of "Pseudowire Type" is defined in [RFC446] along with initial allocated values.

\subsubsection{Frame Relay Specific Interface Parameter Sub-TLV}

A separate document, [RFC4447], describes the PW control and maintenance protocol in detail, including generic interface parameter sub-TLVs. The interface parameter information, when applicable, MUST be used to validate that the PEs and the ingress and egress ports at the edges of the circuit have the necessary capabilities to interoperate with each other. The Interface parameter TLV is defined in [RFC444], and the IANA registry with initial values for interface parameter sub-TLV types is defined in [RFC4446], but the frame relay specific interface parameter sub-TLV types are specified as follows:

- 0x08 Frame Relay Header Length Sub-TLV 
An optional 16-bit value indicating the length of the FR Header, expressed in octets. This OPTIONAL interface parameter Sub-TLV can have value of 2,3 , or 4 , the default being 2 . If this Sub-TLV is not present, the default value of 2 is assumed.

8. Frame Relay Port Mode

The frame relay port mode PW shares the same encapsulation as the HDLC PW and is described in the respective document. [RFC4618]

9. Congestion Control

As explained in [RFC3985], the PSN carrying the PW may be subject to congestion, the characteristics of which depend on PSN type, network architecture, configuration, and loading. During congestion, the PSN may exhibit packet loss that will impact the service carried by the frame relay PW. In addition, since frame relay PWs carry a variety of services across the PSN, including but not restricted to TCP/IP, they may or may not behave in a TCP-friendly manner prescribed by [RFC2914]. In the presence of services that reduce transmission rate, frame relay PWs may thus consume more than their fair share and in that case SHOULD be halted.

Whenever possible, frame relay PWs should be run over trafficengineered PSNs providing bandwidth allocation and admission control mechanisms. IntServ-enabled domains providing the Guaranteed Service (GS) or DiffServ-enabled domains using EF (expedited forwarding) are examples of traffic-engineered PSNs. Such PSNs will minimize loss and delay while providing some degree of isolation of the frame relay PW's effects from neighboring streams.

Note that when transporting frame relay, DiffServ-enabled domains may use AF (Assured Forwarding) and/or DF (Default Forwarding) instead of $E F$, in order to place less burden on the network and to gain additional statistical multiplexing advantage. In particular, if the Committed Information Rate (CIR) of a frame relay VC is zero, then it is equivalent to a best-effort UDP over IP stream regarding congestion: the network is free to drop frames as necessary. In this case, the "DF" Per Hop Behavior (PHB) would be appropriate in a diff-serv-TE domain. Alternatively, if the CIR of a frame relay VC is nonzero and the DE bit is zero in the FR header, then "AF31" would be appropriate to be used, and if the CIR of a frame relay VC is nonzero but the DE bit is on, then "AF32" would be appropriate [RFC3270].

The PEs SHOULD monitor for congestion (by using explicit congestion notification, [VCCV], or by measuring packet loss) in order to ensure that the service using the frame relay PW may be maintained. When a 
$\mathrm{PE}$ detects significant congestion while receiving the $\mathrm{PW}$ PDUs, the BECN bits of the frame relay frame transmitted on the same PW SHOULD be set to notify the remote $\mathrm{PE}$ and the remote frame relay switch of the congestion situation. In addition, the FECN bits SHOULD be set in the FR frames sent out the attachment circuit, to give the FR DTE a chance to adjust its transport layer advertised window, if possible.

If the PW has been set up using the protocol defined in [RFC4447], then procedures specified in [RFC4447] for status notification can be used to disable packet transmission on the ingress PE from the egress $\mathrm{PE}$. The PW may be restarted by manual intervention, or by automatic means after an appropriate waiting time.

10. Security Considerations

PWE3 provides no means of protecting the contents or delivery of the PW packets on behalf of the native service. PWE3 may, however, leverage security mechanisms provided by the MPLS Tunnel Layer. A more detailed discussion of $\mathrm{PW}$ security is given in [RFC3985, RFC4447, RFC3916].

11. Normative References

[RFC4447] Martini, L., Rosen, E., El-Aawar, N., Smith, T., and G. Heron, "Pseudowire Setup and Maintenance Using the Label Distribution Protocol (LDP)", RFC 4447, April 2006.

[RFC4385] Bryant, S., Swallow, G., Martini, L., and D. McPherson, "Pseudowire Emulation Edge-to-Edge (PWE3) Control Word for Use over an MPLS PSN", RFC 4385, February 2006.

[RFC3032] Rosen, E., Tappan, D., Fedorkow, G., Rekhter, Y., Farinacci, D., Li, T., and A. Conta, "MPLS Label Stack Encoding", RFC 3032, January 2001.

[RFC4446] Martini, L., "IANA Allocations for Pseudowire Edge to Edge Emulation (PWE3)", BCP 116, RFC 4446, April 2006.

[RFC4618] Martini, L., Rosen, E., Heron, G., and A. Malis, "Encapsulation Methods for Transport of Point to Point Protocol/High-Level Data Link Control (PPP/HDLC) over Multiprotocol Label Switching (MPLS) Networks", RFC 4618, September 2006 .

[RFC4623] Malis, A. and M. Townsley, "Pseudowire Emulation Edge-toEdge (PWE3) Fragmentation and Reassembly", RFC 4623, August 2006 . 
12. Informative References

[RFC3985] Bryant, S. and P. Pate, "Pseudo Wire Emulation Edge-to-Edge (PWE3) Architecture", RFC 3985, March 2005.

[VCCV] Nadeau, T., et al., "Pseudo Wire Virtual Circuit Connection Verification (VCCV)", Work in Progress, October 2005.

[ATM] Martini, L., et al., "Encapsulation Methods for Transport of ATM Over MPLS Networks", Work in Progress, April 2005.

[RFC4448] Martini, L., Rosen, E., El-Aawar, N., and G. Heron, "Encapsulation Methods for Transport of Ethernet over MPLS Networks", RFC 4448, April 2006.

[FRF1] FRF.1.2, Frame relay PVC UNI Implementation Agreement, Frame Relay Forum, April 2000.

[FRF2] FRF.2.2, Frame relay PVC UNI Implementation Agreement, Frame Relay Forum, April 2002

[RFC3916] Xiao, X., McPherson, D., and P. Pate, "Requirements for Pseudo-Wire Emulation Edge-to-Edge (PWE3)", RFC 3916, September 2004 .

[X36] ITU-T Recommendation X.36, Interface between a DTE and DCE for public data networks providing frame relay, Geneva, 2000 .

[X76] ITU-T Recommendation X.76, Network-to-network interface between public data networks providing frame relay services, Geneva, 2000

[Q922] ITU-T Recommendation Q.922 Specification for Frame Mode Basic call control, ITU Geneva 1995

[Q933] ITU-T Recommendation Q.933 Specification for Frame Mode Basic call control, ITU Geneva 2003

[RFC2914] Floyd, S., "Congestion Control Principles", BCP 41, RFC 2914, September 2000 .

[RFC3270] Le Faucheur, F., Wu, L., Davie, B., Davari, S., Vaananen, P., Krishnan, R., Cheval, P., and J. Heinanen, "MultiProtocol Label Switching (MPLS) Support of Differentiated Services", RFC 3270, May 2002. 
Contributing Author Information

Kireeti Kompella

Juniper Networks

1194 N. Mathilda Ave

Sunnyvale, CA 94089

EMail: kireeti@juniper.net

Giles Heron

Tellabs

Abbey Place

24-28 Easton Street

High Wycombe

Bucks

HP11 1NT

UK

EMail: giles.heron@tellabs.com

Rao Cherukuri

Juniper Networks

1194 N. Mathilda Ave

Sunnyvale, CA 94089

Dimitri Stratton Vlachos

Mazu Networks, Inc.

125 Cambridgepark Drive

Cambridge, MA 02140

EMail: d@mazunetworks.com

Chris Liljenstolpe

Alcatel

11600 Sallie Mae Dr.

9th Floor

Reston, VA 20193

EMail: chris.liljenstolpe@alcatel.com 


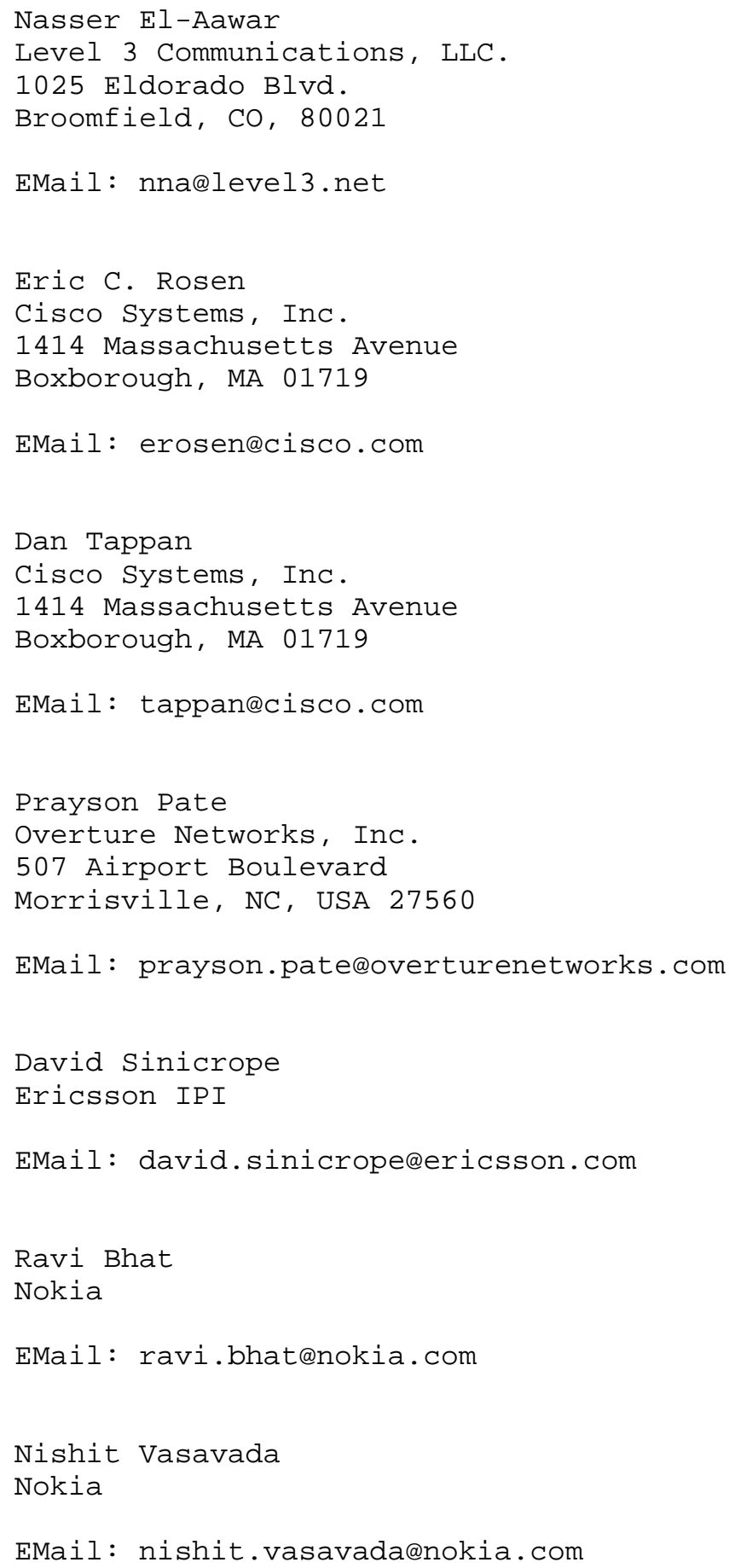




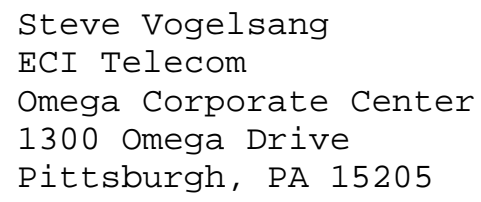

EMail: stephen.vogelsang@ecitele.com

Vinai Sirkay

Redback Networks

300 Holger Way,

San Jose, CA 95134

EMail: sirkay@technologist.com

Authors' Addresses

Luca Martini

Cisco Systems, Inc.

9155 East Nichols Avenue, Suite 400

Englewood, CO, 80112

EMail: lmartini@cisco.com

Claude Kawa

Oz Communications

Windsor Station

1100, de la Gauchetie're St West

Montreal QC Canada

H3B 2S 2

EMail: claude.kawa@oz.com

Andrew G. Malis

Tellabs

1415 West Diehl Road

Naperville, IL 60563

EMail: Andy.Malis@tellabs.com 
Full Copyright statement

Copyright (C) The Internet Society (2006).

This document is subject to the rights, licenses and restrictions contained in BCP 78, and except as set forth therein, the authors retain all their rights.

This document and the information contained herein are provided on an "AS IS" basis and THE CONTRIBUTOR, THE ORGANIZATION HE/SHE REPRESENTS OR IS SPONSORED BY (IF ANY), THE INTERNET SOCIETY AND THE INTERNET ENGINEERING TASK FORCE DISCLAIM ALL WARRANTIES, EXPRESS OR IMPLIED, INCLUDING BUT NOT LIMITED TO ANY WARRANTY THAT THE USE OF THE INFORMATION HEREIN WILL NOT INFRINGE ANY RIGHTS OR ANY IMPLIED WARRANTIES OF MERCHANTABILITY OR FITNESS FOR A PARTICULAR PURPOSE.

Intellectual Property

The IETF takes no position regarding the validity or scope of any Intellectual Property Rights or other rights that might be claimed to pertain to the implementation or use of the technology described in this document or the extent to which any license under such rights might or might not be available; nor does it represent that it has made any independent effort to identify any such rights. Information on the procedures with respect to rights in RFC documents can be found in BCP 78 and BCP 79 .

Copies of IPR disclosures made to the IETF Secretariat and any assurances of licenses to be made available, or the result of an attempt made to obtain a general license or permission for the use of such proprietary rights by implementers or users of this specification can be obtained from the IETF on-line IPR repository at http://www.ietf.org/ipr.

The IETF invites any interested party to bring to its attention any copyrights, patents or patent applications, or other proprietary rights that may cover technology that may be required to implement this standard. Please address the information to the IETF at ietf-ipreietf.org.

Acknowledgement

Funding for the RFC Editor function is provided by the IETF Administrative Support Activity (IASA). 